\title{
TRAFFIC MANAGEMENT STRATEGIES IN AN INTEGRATED SATELLITE/CELLULAR NETWORK FOR MOBILE SERVICES
}

\author{
E. Del Re, P. Iannucci, R. Menolascino, F. Settimo \\ E. Del Re, P. Iannucci: Università di Firenze - Dipartimento di Ingegneria Elettronica, Firenze (ITALY) \\ R. Menolascino, F. Settimo: CSELT - Centro Studi e Laboratori Telecomunicazioni, Torino (ITALY)
}

\begin{abstract}
After a survey on the historical reasons for the current configuration of incompatible terrestrial and satellite mobile networks, the paper compares the classical traffic channel assignment in cellular networks with a more enhanced strategy, suitable to an integrated configuration in that exploits the concept of sharing, peculiar of satellite resources. The impact on network management and planning is pointed out and the results of a simulation model allow a quantitative assessment.The paper also illustrates how the corresponding procedures respect the terrestrial cellular networks protocols.
\end{abstract}

\section{Introductory remarks}

Several elements justify incompatibility of mobile satellite systems with cellular systems, e.g.:

- the coverage area. In North America or Australia, the wider service areas justified regional satellite applications, while national networks have been preferred in Europe. Most current European analogue cellular networks have been developed without a common standard and are impossible to use outside the national borders;

- the customers requirements. Most satellite systems have been tailored on closed user groups specific applications. Terminals and Earth Stations have been designed accordingly and not always interworking with the fixed networks (mandatory in cellular system) is provided;

- the allocated band (UHF for cellular, L-band for satellites). The different radio channel modelling required specific modulation and filtering techniques for radio link optimization. In particular, the grade of service of analogue cellular systems was still considered adequate several years after the introduction of digital technology in the satellite systems;
- the offered services. The early development of message-based satellite systems was supported by digital techniques and deemed commercially appropriate, irrespective of high transmission delays. Voice service has been postponed until voice coding algorithms allowed efficient bandwidth usage. In cellular systems, on the contrary, voice service always had top priority, with data services relegated to a lower rank.

The combination of the above elements produced an incompatibility state until network integration urgency has been perceived. In the literature, e.g. [1], four integration levels, namely: geographic, service, network and system have been identified. Here, system integration is assumed to involve the challenging protocol sharing on the radio interface and the definition of traffic management strategies and procedures is addressed accordingly. A preliminary link budget verification [2], [3] is assumed. Although the methodology is very general, the paper is addressed to the pan European GSM (Global System for Mobile applications) standard. Global coverage and spot beam satellites are absolutely equivalent.

Fig. 1 shows the integrated architecture. A dual mode mobile station (MS), with a common GSM base band section and independent RF units, ensures access to both networks reusing the GSM protocols. The connection between the Fixed Earth Station (FES) and the GSM Switching system (MSC) requires gateway functions in the FES but allows reuse of a GSM Interface. Location Registers interrogation, to achieve MS position and other customer data, is totally specified within the CCITT Signalling System N. 7 and, like network functionalities, is less critical to integrate.

\section{An alternative channel assignment strategy}

\section{1 - Comparison with the cellular networks}

A well known [1] approach to satellite/cellular integration looks at the satellite system as an extension of the GSM network and at satellite beams 
as GSM cells. This implies that traffic channels, irrespective of their nature (cellular or satellite), are allocated to incoming calls following GSM procedures, based on signal strength and quality as received by the MS.

Although it represents a straightforward extension, this behaviour leads to a poor network efficiency [4]. First of all, satellite capacity is strongly limited by frequency or power constraints while frequency reuse "multiplies" GSM channels. Secondly, the sharing degree is much higher for satellite channels than for the GSM ones, only available within cell boundaries: hence, signal-based comparisons between satellite and GSM channels are not fair. These considerations should discourage to allocate improperly a satellite channel to an incoming call, i.e. when at least one GSM channel is available in the originating cell, the following selective call set up procedure is suggested:

try first to allocate a GSM traffic channel; only in case of a blocking state on the serving GSM cell, try call set up on a satellite channel.

This strategy discriminates channel types on the base of network criteria (here, resource congestion), not simply on signal comparison. In traffic theory terms, satellite channels represent an overflow possibility to the GSM ones that, being more accessible, play the role of primary channels.

\section{2 - Application scenarios}

An enhanced traffic management strategy in an integrated network helps to overcome the classical satellite roles of complementing the GSM network in uncovered areas and of supplying purely additional channels in common regions. As a matter of fact, the GSM fixed traffic channel capacity is a severe limitation when traffic expectations are exceeded. Two typical situations are encountered both periodically and during subscribers growth and belong to Network Management and Planning issues.

The first situation follows from users mobility, an additional uncertainty component to the random voice traffic process that can cause focused overloads. Cellular networks are vulnerable to such changes in the traffic patterns, because channel dimensioning is normally tailored on peak hour requirements and can be changed only with complex dynamic management procedures. Complexity regards: traffic increase detection, channel dimensioning evaluation and, possibly, redefinition of a carrier-to-cell plan that respects frequency reuse. Satellite channels, used as overflow resources, are capable of absorbing the cellular traffic peaks and this feature allows both to dimension the GSM channels according to looser constraints; and to avoid intervention of periodic reconfigurations.

The customers growth, currently experienced by cellular networks produces an average increase of the offered traffic. To cope with this problem, the GSM networks employ cell splitting, cell radius reduction, increase of carriers per cell, i.e. actions resulting in infrastructure costs, due to additional RF equipment and/or sites installation. In this process, a satellite can support the GSM network planning, since the investments due to customers growth can be delayed. More consistent advantages can be achieved with a traffic handling strategy applied to the "additional" satellite channels.

\section{3 - An application example}

A comparison between two (conventional and enhanced) channel assignment strategies has been performed on an example of 44 GSM cells with an overlapping satellite spot beam. Three classes of terrestrial cells incorporate traffic and propagation conditions typically encountered in urban, suburban and rural environments. For each class the allocated traffic channels ensure a nominal (no supporting satellite system) blocking probability equal to 0.03 .

Some marginal traffic (customers roaming outside the GSM coverage but still in the satellite beam) is assumed: such customers can access only and directly the satellite channels, again with a blocking probability equal to 0.03 . (Hence, if the GSM and the satellite networks were independent, they would offer the same quality of service). The average conversation time is 2 minutes and, as usual, the offered traffic is supposed poissonian.

With the conventional strategy, a dual mode MS will select a satellite channel instead of a GSM one whenever signal quality is better. Different satellite visibilities have been modelled with a set

$$
S_{\mathrm{p}}=\left\{\mathbf{P}_{1}, \mathbf{P}_{2}, \mathbf{P}_{3}\right\}
$$

of three probability values (one for each cell class). The i-th $(\mathrm{i}=1-3)$ value of $S_{\mathbf{p},}\left[\mathbf{P}_{1}\left(\mathbf{P}_{2}, \mathbf{P}_{3}\right)\right.$ for rural (suburban, urban) cells] represents the probability of selecting a satellite channel in an i-th class cell even when free GSM channels exist. 
Figs 2 - 4 show the behaviour of some major performance figure, as perceived by mobile users. The System Blocking Probability (Fig. 3) represents the probability that a call is rejected because no traffic channel can be allocated. This happens:

- for conventional call set up, if either the serving GSM cell or the satellite capacity, depending on the selected network, is blocked (no traffic channel available);

- for selective call set up, if the satellite capacity is blocked. A blocking state on the GSM channels pool does not necessarily mean call rejection if there are free satellite channels.

The solid line in Fig. 2 indicates the performance achievable with selective call set up; the three dotted lines indicate, for three different $S_{p}$ triplets, the achievable performance with conventional set up. It is pointed out that the overflow capability reduces the blocking probability of about $30 \%$ and, secondly that with no overflow, the performance of the integrated network is rather poor. If the triplets values were optimistic, the probability of choosing improperly a satellite channel would be higher, with performance even worse.

Fig. 3 shows the GSM Network blocking probability, i.e. the probability of rejecting a call originating in the GSM network. This happens:

- with conventional call set up, if the serving cell (selected by the MS) is blocked;

- with selective call set up, if both the GSM serving cell and the satellite are blocked.

Additional satellite channels always reduce the nominal GSM Network blocking probability (0.03). Nevertheless, in the former case (dotted lines), the fixed partitioning of the satellite channels makes the satellite capacity practically ininfluent on the resulting blocking probability. It is noted that for selective call set up (solid line) the GSM network performance improves even if the marginal satellite traffic increases (increased satellite capacity). This occurs because high capacity channel pools have a higher traffic handling capability.

The benefits achieved on both System and GSM Network blocking probabilities are compensated (Fig. 4) by a worsening of the Satellite Blocking Probability, perceived by mobile users when either roam in marginal areas or are better served by a satellite channel. It is expressed by the fraction of calls, accessing directly the satellite network, that result blocked. Again, the advantage of selective call set up (solid line) is evident, although the overall figure is poor, even for high satellite capacity values. An improvement possibility is a partitioning of the satellite channels pool, so that only a preassigned fraction can serve the GSM overflow traffic.

\section{4 - Handover}

In cellular networks, handover procedures prevent from call disconnections due to excessive signal degradation in cell transition. In an integrated satellite/GSM network, the concept can include the passage of ongoing conversations from radio channels belonging to different networks. In principle, two handover types, GSM-to-satellite and satellite-toGSM, exist.

The former can occur when a busy MS leaves the GSM coverage but (at least) one free satellite channel still supplies an acceptable signal quality. Hence they are a pure GSM handovers extension and can be a desirable feature to maintain call continuity; however, they are not traffic management tools and hence are left out of the paper.

Satellite-to-GSM handovers, on the contrary, aim at enhancing the network efficiency by maximizing the availability (i.e. the sharing opportunity) of satellite channels. Such a functionality is closely related to the selective call set up procedure (sect. 2.1) and consists in:

checking the occupancy state of the GSM channels that could be allocated to an ongoing satellite conversation; if at least one free channel exists in a suitable GSM cell, the call is handed over to the GSM network.

Although satellite-to-GSM handovers have not been included in the performance model, a further reduction of the satellite and system blocking probabilities is expected.

\section{Procedure description}

\section{1 - Selective call set-up}

To maximise resource efficiency in the integrated system, dual mode MSs should access the satellite network when either they are in an area without GSM coverage (not implemented or temporarily 
unavailable) or the serving cell is blocked for traffic overload.

Two different procedures have to be used. In the first case, the MS can detect the terrestrial network unavailability, scanning GSM carriers: if no other GSM cell ensures an acceptable signal quality, the MS may try establishing the call on the satellite link. When, on the contrary, the serving cell is blocked, the integrated set-up procedure is more complex since the MS cannot determine the cell state at call establishment. Hence access to the satellite network can only occur as a second attempt, after failure on the terrestrial network. Two situations must be distinguished:

a)The called mobile terminal detects a blocking state in the serving cell. The terrestrial network must ensure an alternative routing (currently not foreseen in the GSM network) to the FES; the associated procedure would require some protocol enhancement but not on the GSM radio interface and, hence, not too critical. The MS ignores which system will establish the communication and must be ready to receive a call warning from (and exchange service messages with) both systems. It is technically feasible at the expense of MS components duplication.

b) The calling mobile terminal detects a blocking state in the serving cell. All necessary activities can be undertaken by the mobile terminal, simplifying attempts to establish the call through the satellite link. The main problem turned out to be the long time interval for procedure completion, that includes the GSM set-up failure; the spot beam selection, the mobilelocation updating in the satellite network and the set-up on the satellite link.

\section{2 - Satellite-to-GSM handover procedure}

With an ongoing satellite conversation, the decision to start a handover towards a GSM cell must be taken by the satellite system. The most reasonable criteria seem the following:

a) the satellite network looks for a free GSM channel as soon as the occupancy state exceeds a given threshold;

b) the call is immediately handed over, as soon as a GSM channel is available in a suitable cell.

It is recalled that in the GSM system, a busy mobile measures the received signals relating to six adjacent cells and transmits the results to the base station; when signal degradation is excessive, the network decides the cell where the conversation can be handed over. Consequently, a satellite-to-GSM handover procedure might be organized as follows:

1) after call set-up, the mobile terminal informs the satellite earth station the identification code of the serving GSM cell;

2) the fixed earth station transmits the list of the GSM carriers to be monitored by the mobile;

3) the terminal reports the related results specifying the complete GSM identity of the cell with the strongest signal, i.e. the serving cell;

4) the list of carriers to transmit is updated by the satellite station according to the terminal location;

5) When it is needed, the handover procedure may be executed according to the GSM standard.

\section{Conclusions}

The market size of mobile applications recommends to devote consistent efforts to the definition of new architectures (e.g. UMTS) but also to explore the opportunities offered by integrating the existing networks. Traffic management is a promising investigation area provided that satellite peculiarities are fully exploited, so that satellite networks truly integrate, not only complement, GSM networks. Common procedures, grounded on GSM specs, will speed up the integration process with benefits to mobile users and to PLMN Operators.

\section{R E F E R E N C ES}

[1] A. Arcidiacono - Integration between Terrestrialbased and Satellite-based Land Mobile Communication Systems - IMSC '90, Ottawa, June $17-20,1990$

[2] F. Delli Priscoli, F. Muratore - Integration Study between the GSM System and a satellite system operating at L-band - Paper submitted to ICC ' 93

[3] L. Bonzano, M. Carosi, F. Delli Priscoli, F. Muratore - Study on the integration between cellular network and satellite system - Paper submitted to INFOCOM ' 93

[4] F. Delli Priscoli, E. Del Re, R. Menolascino, I. Mistretta, F. Settimo - Issues for the integration of satellite and terrestrial cellular networks for mobile communications - Workshop on Advanced Network and Technology Concepts for Mobile, Micro and Personal Communications, Pasadena, 1991

Work partially supported by MURST and ASI 


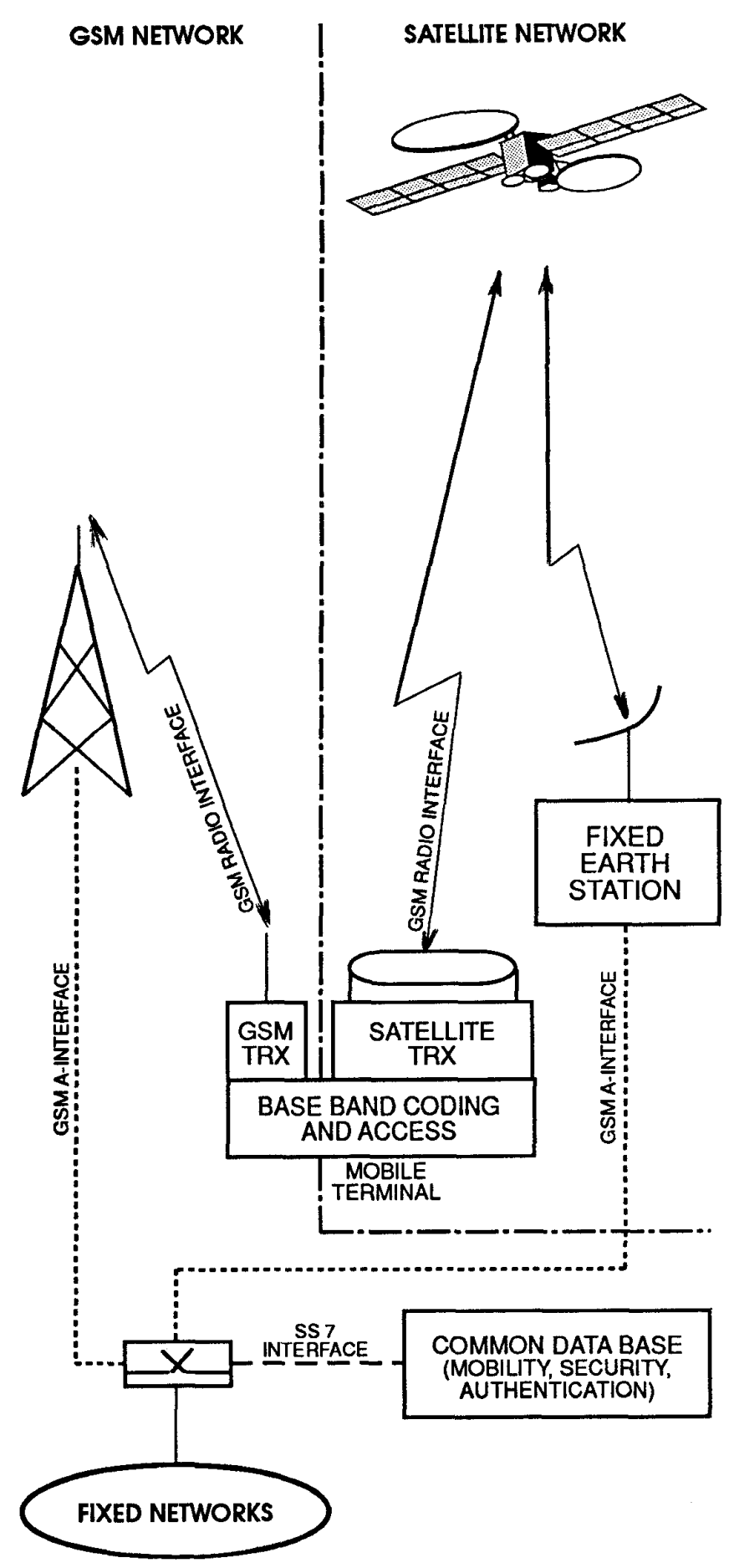

Fig. 1) System Integration

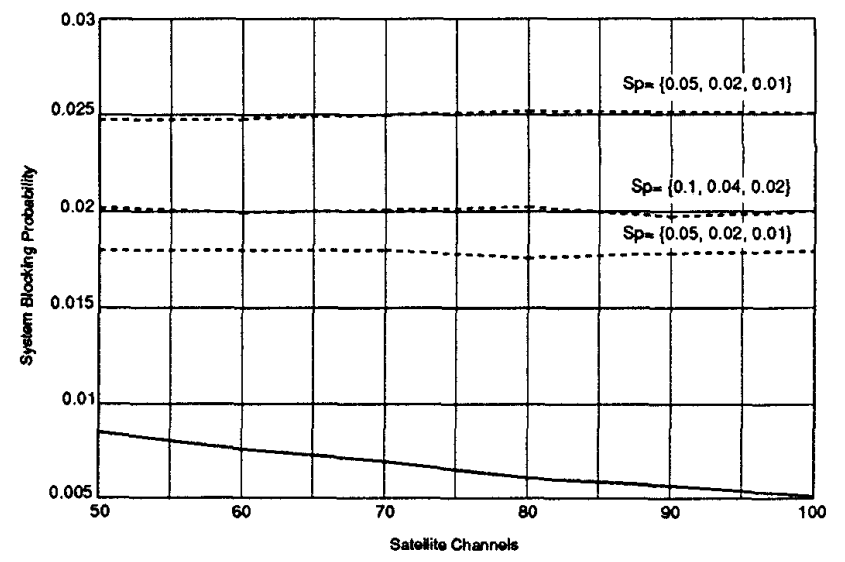

Fig. 2) System Blocking Probability

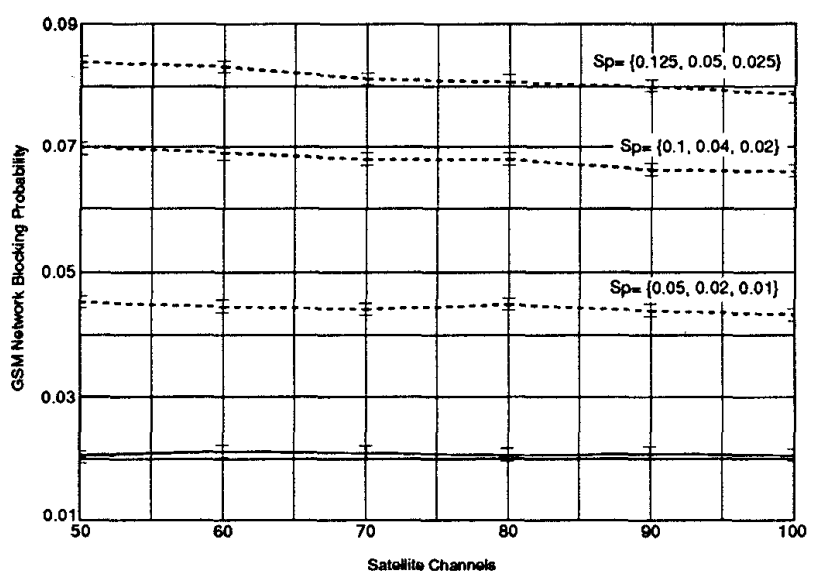

Fig. 3) GSM Network Blocking Probability

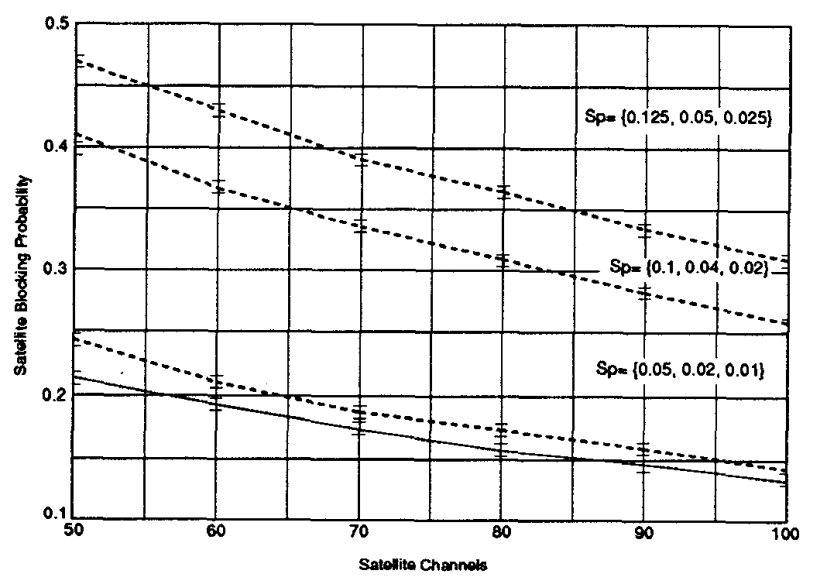

Fig. 4) Satellite Blocking Probability 